\title{
Multi-patch nonsingular isogeometric boundary element analysis in 3D
}

\author{
Y.J. Wang ${ }^{\mathrm{a}}$, D.J. Benson ${ }^{\mathrm{a}, *}$ \\ ${ }^{a}$ Department of Structural Engineering, University of California, San Diego, La Jolla, USA
}

\begin{abstract}
A multi-patch nonsingular isogeometric boundary element method (IGABEM) for 3D problems is presented that provides accurate solutions for multi-patch IGABEM. In order to conveniently implement this method, based on the Greville abscissae, a new collocation method moves the first and the last collocation points of each parametric direction inside of their patches, and a simple method for merging equations handles the extra equations. The numerical results verify the accuracy and efficiency of the present method by comparing it to the conventional IGABEM.
\end{abstract}

Keywords: Isogeometric Analysis, Boundary element method, Nonsingular integration, Multi-patch, elastostatics

\section{Introduction}

Isogeometric analysis (IGA) [1,2] uses the same basis functions for the geometric modeling and computational analysis. After over a decade of continuous development, IGA has become one of the most efficient methods for bridging the gap between computer-aided design (CAD) and engineering analysis. The initial implementations of IGA used non-uniform rational B-splines (NURBS) to address a broad range of applications, including structural vibration [3], fluids [4, 5], fluid-structure interaction [6-8], cables and shells [9-12], contact [13, 14], fracture [15], shape optimization [16, 17], topology optimization [18, 19]. Although NURBS is a standard technology in CAD and IGA, there are a growing number of new spline technologies supporting local refinement that have been recently used

${ }^{*}$ Corresponding author. E-mail: dbenson@ucsd.edu; Phone: +1 8585345928 
in IGA, including T-splines [20, 21], PHT-splines [22] hierarchical B-splines or NURBS [23, 24] and Powell-Sabin splines [25, 26].

While the FEM-IGA has achieved great success and has attracted most of the IGA researchers, a remaining critical challenge is how to efficiently and exactly parameterize a volumetric domain from its boundary [27, 28]. Three dimensional problems need trivariate NURBS solids for analysis but CAD systems use a boundary representation where only bivariate NURBS surface is available. In order to solve this problem, the boundary element method (BEM) [29, 30] was applied to IGA for several classical partial differential equations, e.g. Laplace equation [31], elastostatics [32, 33], potential flow [34], Helmholtz problem [35] and wave-resistance problem [36]. The isogeometric BEM is called IGABEM for short in this paper.

Unlike the FEM, the BEM uses the fundamental solutions to construct boundary integral equations (BIEs) to solve boundary value problems. The fundamental solutions are usually of the type $O\left(1 / r^{\alpha}\right)$, resulting in nearly singular and singular integrals that cannot be accurately evaluated by standard Gaussian quadrature. Accurate and efficient computation of these nearly singular and singular integrals plays a crucial role in the IGABEM as well as in the BEM. For singular integrals $(r=0)$, the proposed methods include, but are not limited to, analytical methods [37, 38], degenerate mapping methods [39], radial integration methods [40, 41] and non-linear transformation methods [42-44]. For nearly singular integrals ( $r$ is very close to 0 ), an equally broad range of methods are available, including domain division methods $[45,46]$, semi-analytical and analytical methods [47, 48], and a series of transformation methods [49-55].

Regardless of which singularity-removal method is used, the complexity of the implementation and the computational cost will increase to a certain degree. Fortunately, careful studies of the BIE formulations have revealed that the BIEs can be recast in weakly-singular, or even nonsingular forms. Cruse [56] created a weakly-singular form by expressing the free term coefficient matrix $C_{i j}$. Cruse and Aithal [57] developed a nonsingular BIE using Taylor series expansions, but some terms of the integrands are still nearly singular. Liu et al. [58, 59] proposed several identities for the fundamental solutions and applied them to weakly-singular BEM to derive nonsingular BIEs for potential and elastostic problems [60]. These nonsingular forms need to compute the derivatives of the boundary conditions (e.g. potential, displacement), some of which are unknown and not easy to obtain. In recent years, a new nonsingular BEM was presented by Klaseboer et al. [61, 62]. Heltai et al. [63] extended the work of Klaseboer et al. to the single-patch IGA for 3D Stokes flows, and demonstrated the validity of the 
nonsingular IGABEM.

In this paper, representations of the displacement derivatives and stresses in 3D elastostatics from the boundary displacements and tractions are derived, and they are combined with the nonsingular BEM and IGA to implement a multi-patch nonsingular IGABEM. The paper is organized as follows: Section 2 provides a brief overview of NURBS fundamentals. Section 3 summarizes the boundary integral equations for 3D elastostatics. Section 4 presents the nonsingular IGABEM and its implementation in detail. Section 5 provides numerical examples to demonstrate the efficiency and accuracy of the nonsingular IGABEM. Finally, conclusions and future research is proposed in Section 6.

\section{An overview of NURBS fundamentals}

Non-uniform rational basis splines (NURBS) are commonly used in computeraided design (CAD) and computer graphics (CG) for generating and representing curves and surfaces [64]. NURBS are constructed from B-splines. The coefficients of these basis functions are points in physical space, referred to as control points, determine the shape of the spline curve. A knot vector $\Xi$, which determines where and how the control points affect the curve, is a sequence of non-decreasing real numbers representing parametric coordinates of the curve:

$$
\Xi=\left\{\xi_{1}, \xi_{2}, \ldots, \xi_{n+p+1}\right\}
$$

where $p$ is the order of the $\mathrm{B}$-spline and $n$ is the number of basis functions constructing the B-spline curve. The interval $\left[\xi_{1}, \xi_{n+p+1}\right]$ is called a patch, and the knot interval $\left[\xi_{i}, \xi_{i+1}\right]$ is called a span.

Given a knot vector, the B-spline basis functions are defined recursively for zero order $(p=0)$,

$$
B_{i, p}(\xi)= \begin{cases}1 & \text { if } \xi_{i} \leq \xi<\xi_{i+1} \\ 0 & \text { otherwise }\end{cases}
$$

and for non-zero order $(p>0)$

$$
B_{i, p}(\xi)=\frac{\xi-\xi_{i}}{\xi_{i+p}-\xi_{i}} B_{i, p-1}(\xi)+\frac{\xi_{i+p+1}-\xi}{\xi_{i+p+1}-\xi_{i+1}} B_{i+1, p-1}(\xi) .
$$

Given a control net consisting of the control points $\boldsymbol{P}_{i, j}$, a bivariate B-spline surface is obtained as the tensor product of two B-spline curves with knot vectors 


$$
\begin{aligned}
& \Xi=\left\{\xi_{1}, \xi_{2}, \ldots, \xi_{n+p+1}\right\} \text { and } \mathcal{H}=\left\{\eta_{1}, \eta_{2}, \ldots, \eta_{m+q+1}\right\} \\
& \qquad S(\xi, \eta)=\sum_{i=1}^{n} \sum_{j=1}^{m} B_{i, p}(\xi) B_{j, q}(\eta) \boldsymbol{P}_{i, j},
\end{aligned}
$$

where $B_{i, p}(\xi)$ and $B_{j, q}(\eta)$ are univariate B-spline basis functions of order $p$ and $q$, corresponding to knot vectors $\Xi$ and $\mathcal{H}$. The patch for the surface is now the domain $\left[\xi_{1}, \xi_{n+p+1}\right] \times\left[\eta_{1}, \eta_{m+q+1}\right]$.

NURBS basis functions are obtained from B-splines by assigning a positive weight $w_{i}$ to each basis function:

$$
N_{i, p}(\xi)=\frac{B_{i, p}(\xi) w_{i}}{\sum_{j=1}^{n} B_{j, p}(\xi) w_{j}},
$$

A NURBS surface of order $p$ in the $\xi$ direction and order $q$ in the $\eta$ direction is a bivariate piecewise rational function of the form

$$
S(\xi, \eta)=\sum_{i=1}^{n} \sum_{j=1}^{m} \frac{B_{i, p}(\xi) B_{j, q}(\eta) w_{i, j} \boldsymbol{P}_{i, j}}{\sum_{k=1}^{n} \sum_{l=1}^{m} B_{k, p}(\xi) B_{l, q}(\eta) w_{k, l}},
$$

where $w_{i, j}$ is the weight value corresponding to the tensor product $B_{i, p}(\xi) B_{j, q}(\eta)$. If all weights are set to 1, Eq. (6) has the same form as Eq. (4).

Introducing the piecewise rational basis functions

$$
N_{i, j}(\xi, \eta)=\frac{B_{i, p}(\xi) B_{j, q}(\eta) w_{i, j} \boldsymbol{P}_{i, j}}{\sum_{k=1}^{n} \sum_{l=1}^{m} B_{k, p}(\xi) B_{l, q}(\eta) w_{k, l}},
$$

the surface Eq.(6) may be written in the familiar shape function notation as

$$
S(\xi, \eta)=\sum_{i=1}^{n} \sum_{j=1}^{m} N_{i, j}(\xi, \eta) \boldsymbol{P}_{i, j}
$$

Some of the important properties of the function $N_{i, j}(\xi, \eta)$ are summarized as below:

1. Nonnegativity: $N_{i, j}(\xi, \eta) \geq 0$ for all $i, j, \xi$ and $\eta$;

2. Partition of unity: $\sum_{i=1}^{n} \sum_{j=1}^{m} N_{i, j}(\xi, \eta)=1$ for all $(\xi, \eta)$;

3. Local support: if $(\xi, \eta)$ is outside the span domain of $\left[\xi_{i}, \xi_{i+p+1}\right) \times\left[\eta_{j}, \eta_{j+q+1}\right)$, $N_{i, j}(\xi, \eta)=0$

4. Continuity: the interior of knot span domain is continuous up to $C^{\infty}$, and the continuity between knot spans are $C^{p-k}$ and $C^{q-e}$ where $k$ and $e$ are the multiplicity of the knots in the $\xi$ and $\eta$ directions, respectively. 


\section{Boundary integral formulation}

The summation convention is used for repeated lower-case Latin indices in the remainder of the paper, where the summation is carried out over the range of the repeated subscript unless explicitly indicated otherwise.

For a physical domain $\Omega$ with a boundary $\Gamma$, in the absence of body forces, the discrete form boundary integral equation (BIE) for linear elastostatics is formulated as

$$
C_{i j}(s) u_{j}(s)+\int_{\Gamma} T_{i j}(s, \boldsymbol{x}) u_{j}(\boldsymbol{x}) d \Gamma(\boldsymbol{x})=\int_{\Gamma} U_{i j}(s, \boldsymbol{x}) t_{j}(\boldsymbol{x}) d \Gamma(\boldsymbol{x}),
$$

where $s$ is a source point (collocation point), $\boldsymbol{x}$ is a field point (integration point), $u_{j}(\boldsymbol{x})$ and $t_{j}(\boldsymbol{x})$ are the $j$-th component of the displacement and the traction at field point $x, C_{i j}(s)$ is the free term coefficient depending on the boundary geometry at the source point $s$, and $U_{i j}(s, x)$ and $T_{i j}(s, x)$ are the fundamental solution kernels for $3 \mathrm{D}$ problems which are given as

$$
\begin{aligned}
U_{i j}(s, \boldsymbol{x}) & =\frac{1}{16 \pi \mu(1-v) r}\left\{(3-4 v) \delta_{i j}+r_{, i} r_{, j}\right\}, \\
T_{i j}(s, \boldsymbol{x})= & -\frac{1}{8 \pi(1-v) r^{2}}\left\{\frac{\partial r}{\partial \boldsymbol{n}}\left[(1-2 v) \delta_{i j}+3 r_{, i} r_{, j}\right]\right. \\
& \left.-(1-2 v)\left(r_{, i} n_{, j}-r_{, j} n_{, i}\right)\right\},
\end{aligned}
$$

where $r=|\boldsymbol{x}-\boldsymbol{s}|$ is the distance between the field point and the source point, $r_{, i}=\frac{\partial r}{\partial x_{i}}$ is the partial derivative in the $i$-th direction, $n_{i}$ is the $i$-th component of the unit outward normal $n, v$ is Poisson's ratio, $\mu$ is shear modulus, and $\delta_{i j}$ is the Kronecker delta.

If the boundary is discretized into $E$ elements and each element contains $A$ nodes, then the discrete form of Eq. (9) is

$$
C_{i j}(s) u_{j}(s)+\sum_{e=1}^{E} \sum_{\alpha=1}^{A} u_{j}^{e \alpha}(\boldsymbol{x}) H_{i j}^{e \alpha}(s, \boldsymbol{x})=\sum_{e=1}^{E} \sum_{\alpha=1}^{A} t_{j}^{e \alpha}(\boldsymbol{x}) G_{i j}^{e \alpha}(s, \boldsymbol{x}),
$$

in which $u_{j}^{e \alpha}(x)$ and $t_{j}^{e \alpha}(x)$ are the $j$-th component of the displacement and traction of the $\alpha$-th node of element $e$, and

$$
H_{i j}^{e \alpha}(s, \boldsymbol{x})=\int_{\Gamma_{e}} T_{i j}(s, \boldsymbol{x}) N^{e \alpha}(\boldsymbol{x}) d \Gamma(\boldsymbol{x}),
$$




$$
G_{i j}^{e \alpha}(s, \boldsymbol{x})=\int_{\Gamma_{e}} U_{i j}(s, \boldsymbol{x}) N^{e \alpha}(\boldsymbol{x}) d \Gamma(\boldsymbol{x}),
$$

where $\Gamma_{e}$ is the domain of element $e$, and $N^{e \alpha}(x)$ is the shape function of the $\alpha$-th node of element $e$ at point $\boldsymbol{x}$.

Collocating source points (nodes) on the boundary and applying Eq. (12) at each source point, by merging the corresponding coefficients, the matrix form of the BIE system can be assembled as

$$
H u=G t
$$

note that the entries of matrix $\boldsymbol{H}$ are comprised of $C_{i j}(s)$ in Eq. (12) and $H_{i j}^{e \alpha}(s, \boldsymbol{x})$ in Eq. (13).

Assigning all unknown displacements and tractions to the left hand side, and other known ones to the right hand side, Eq. (15) can be reassembled to the familiar linear algebraic equation system

$$
\boldsymbol{A q}=\boldsymbol{b}
$$

where $\boldsymbol{q}$ is the vector containing the unknown $u$ and $t$ components, and $\boldsymbol{A}$ is the associated collection of coefficients from $\boldsymbol{H}$ and $\boldsymbol{G}$.

\section{Non-singular IGABEM for three-dimensional elastostatics}

The fundamental solution kernels $U_{i j}(s, \boldsymbol{x})$ and $T_{i j}(\boldsymbol{s}, \boldsymbol{x})$ in Eqs. (10) and (11)

contain terms proportional to $\frac{1}{r}$ and $\frac{1}{r^{2}}$ respectively. These terms tend to infinity when the distance between the source point and the field point tend to $0(r \rightarrow 0)$, which causes the singularity of Eqs. (14) and (13). When $r$ equals 0, Eq. (14) is a strongly singular integral and Eq. (13) is a weakly singular integral; when $r$ is very close to $0(r \neq 0)$, both of Eqs. (14) and (13) are nearly singular integrals. In the conventional BEM, various singularity-removal methods are used to evaluate these singular integrals [37-55] to achieve accurate analysis results. Instead of using these methods, a more accurate and efficient nonsingular BEM is adopted and applied to IGA in this paper.

\subsection{The IGABEM}

The major difference between the IGABEM and the conventional BEM is that the summation $\sum_{\alpha=1}^{A}$ in Eq. (12) involves the control points instead of nodes. In 
the parametric coordinate system $(\xi, \eta)$, the displacements and tractions at point $\boldsymbol{x}(\xi, \eta)$ are evaluated from the control point values using the formula

$$
\begin{aligned}
& u_{i}(\xi, \eta)=\sum_{\alpha=1}^{A} N_{\alpha}(\xi, \eta) u_{i}^{\alpha}, \\
& t_{i}(\xi, \eta)=\sum_{\alpha=1}^{A} N_{\alpha}(\xi, \eta) t_{i}^{\alpha},
\end{aligned}
$$

where $\alpha$ is the $\alpha$-th control point of the element.

A comparison between isogeometric elements and Lagrange elements is shown in Figure 1, with a patch containing 4 quadratic elements (order $p=2$ ), and both the knot vectors in $\xi$ and $\eta$ are $[0,0,0,0.5,1,1,1]$. The left-bottom element is the objective element. In the isogeometric element, some of the control points are outside the element (in general, the control points are not necessarily in the problem domain) and the continuity between the elements can reach $C^{p-1}\left(C^{1}\right.$ for the current example). In the Lagrange element, all nodes are in the element domain, and the continuity between the elements is always $C^{0}$ no matter the degree of elements. Another notable difference is that the total number of control points in the isogeomeric mesh is much less than the number of nodes in the Lagrange mesh (16 vs. 25 in this example), meaning that the number of equations for IGABEM is much smaller than for the traditional BEM for the same size, degree, and number elements.

\subsection{The nonsingular IGABEM formulation}

\subsubsection{Nonsingular BIE}

In the BIE Eq. (9), the free term coefficient $C_{i j}(s)$ may be written as

$$
C_{i j}(s)=-\int_{\Gamma} T_{i j}(s, x) d \Gamma(x) .
$$

Applying the above equation in Eq. (9), the following weakly-singular form of the BIE is obtained

$$
\int_{\Gamma} T_{i j}(s, \boldsymbol{x})\left[u_{j}(\boldsymbol{x})-u_{j}(\boldsymbol{s})\right] d \Gamma(\boldsymbol{x})=\int_{\Gamma} U_{i j}(\boldsymbol{s}, \boldsymbol{x}) t_{j}(\boldsymbol{x}) d \Gamma(\boldsymbol{x}) .
$$

Using the fourth identity for the elastostatic problems [60],

$$
\int_{\Gamma} T_{i j}(s, \boldsymbol{x})\left(x(\boldsymbol{x})_{k}-x(\boldsymbol{s})_{k}\right) d \Gamma(\boldsymbol{x})=E_{j k p q} \int_{\Gamma} U_{i p}(\boldsymbol{s}, \boldsymbol{x}) n_{q}(\boldsymbol{x}) d \Gamma(\boldsymbol{x}),
$$




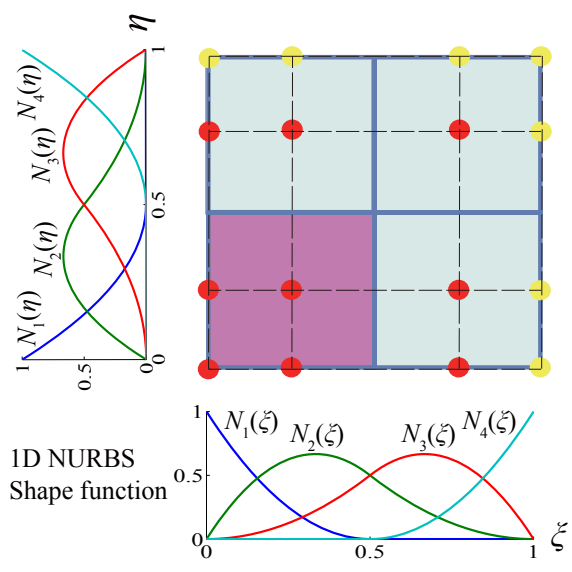

(a) Isogeometric element

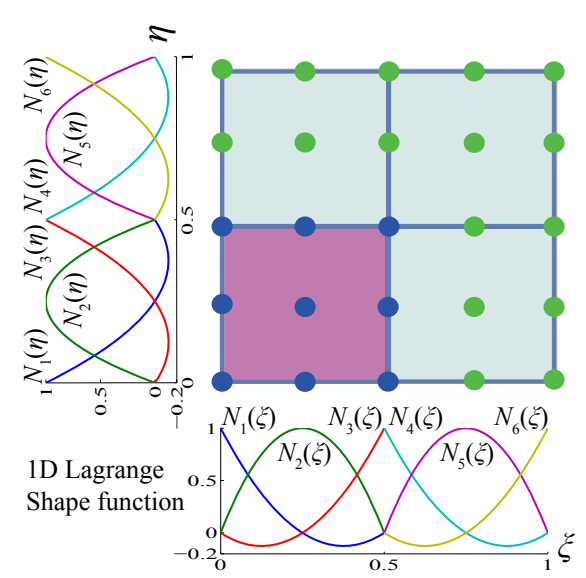

(b) Lagrange element

Figure 1: Isogeometric element vs. Lagrange element for a $1 \times 1$ single-patch surface model. Quadratic elements are used. Red points are the control points of the purple isogeometric element, and blue points are the nodes of the purple Lagrange element.

the resulting formulation is

$$
\int_{\Gamma} T_{i j}(s, \boldsymbol{x}) u_{j, k}(\boldsymbol{s})\left(x(\boldsymbol{x})_{k}-x(\boldsymbol{s})_{k}\right) d \Gamma(\boldsymbol{x})=E_{j k p q} u_{j, k}(s) \int_{\Gamma} U_{i p}(\boldsymbol{s}, \boldsymbol{x}) n_{q}(\boldsymbol{x}) d \Gamma(\boldsymbol{x}),
$$

where $x(\boldsymbol{x})_{k}$ and $x(s)_{k}$ are the $k$-th components of the physical coordinates at $\boldsymbol{x}$ and $s$ respectively, $n_{q}(\boldsymbol{x})$ is the $q$-th component of the outward normal at $\boldsymbol{x}$, and $E_{j k p q}$ is the elastic modulus tensor.

Subtracting Eq. (22) from Eq. (20) gives the nonsingular form of the BIE,

$$
\begin{aligned}
& \int_{\Gamma} T_{i j}(s, \boldsymbol{x})\left[u_{j}(\boldsymbol{x})-u_{j}(\boldsymbol{s})-u_{j, k}(\boldsymbol{s})\left(x(\boldsymbol{x})_{k}-x(\boldsymbol{s})_{k}\right)\right] d \Gamma(\boldsymbol{x}) \\
& =\int_{\Gamma} U_{i j}(\boldsymbol{s}, \boldsymbol{x})\left[t_{j}(\boldsymbol{x})-E_{j k p q} u_{j, k}(\boldsymbol{s}) n_{q}(\boldsymbol{x})\right] d \Gamma(\boldsymbol{x}) .
\end{aligned}
$$

Due to $E_{j k p q} u_{j, k}=E_{p q j k} u_{j, k}=\sigma_{p q}$, the above formulation can be expressed as

$$
\begin{aligned}
& \int_{\Gamma} T_{i j}(s, \boldsymbol{x})\left[u_{j}(\boldsymbol{x})-u_{j}(\boldsymbol{s})-u_{j, k}(\boldsymbol{s})\left(x(\boldsymbol{x})_{k}-x(\boldsymbol{s})_{k}\right)\right] d \Gamma(\boldsymbol{x}) \\
& =\int_{\Gamma} U_{i j}(\boldsymbol{s}, \boldsymbol{x})\left[t_{j}(\boldsymbol{x})-\sigma_{j k}(\boldsymbol{s}) n_{k}(\boldsymbol{x})\right] d \Gamma(\boldsymbol{x})
\end{aligned}
$$

which is equivalent to the nonsingular form of BIE in [60] and more convenient for implementation. The key issue associated with using Eq. (24) is how to evaluate $u_{j, k}(s)$ and $\sigma_{j k}(s)$ from the displacements and tractions. 


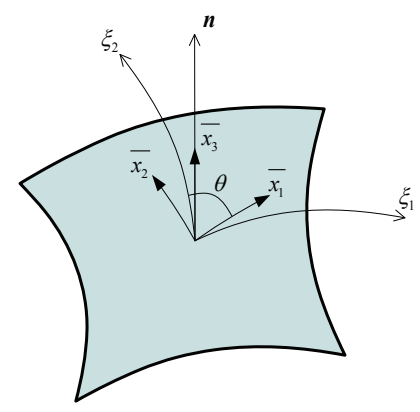

Figure 2: Local orthogonal system for evaluating the derivatives of displacements.

\subsubsection{Stress evaluation}

The traction-recovery method [45] is modified to evaluate boundary stresses in the current IGABEM formulation. Following their development, a local Cartesian coordinate system $\bar{x}_{i}$ is defined as shown in Figure 2, where $\bar{x}_{1}$ and $\bar{x}_{2}$ are tangential to the surface and $\bar{x}_{3}$ is directed in the outward normal direction. In order to concisely describe the formulation, the notation $\left(\xi_{1}, \xi_{2}\right)$ replaces $(\xi, \eta)$ for the parametricparametric coordinates.

The derivatives of the parametric coordinates are

$$
\frac{\partial \xi_{1}}{\partial \bar{x}_{1}}=\frac{1}{m_{1}}, \frac{\partial \xi_{1}}{\partial \bar{x}_{2}}=\frac{-\cos \theta}{\left|m_{1}\right| \sin \theta}, \frac{\partial \xi_{2}}{\partial \bar{x}_{1}}=0, \frac{\partial \xi_{2}}{\partial \bar{x}_{2}}=\frac{1}{\left|m_{2}\right| \sin \theta},
$$

where $m_{k}$ and the angle $\theta$ are given as

$$
\begin{aligned}
& \left|m_{k}\right|=\sqrt{\left(\frac{\partial x_{1}}{\partial \xi_{k}}\right)^{2}+\left(\frac{\partial x_{2}}{\partial \xi_{k}}\right)^{2}+\left(\frac{\partial x_{3}}{\partial \xi_{k}}\right)^{2}}, \\
& \cos \theta=\frac{1}{\left|m_{1}\right|\left|m_{2}\right|} \frac{\partial x_{i}}{\partial \xi_{1}} \frac{\partial x_{i}}{\partial \xi_{2}} .
\end{aligned}
$$

The local displacements and tractions are expressed in terms of the global displacements and tractions

$$
\bar{u}_{i}=L_{i j} u_{j}, \bar{t}_{i}=L_{i j} t_{j},
$$

in which $L_{i j}$ are entries of the rotation matrix $\boldsymbol{L}$

$$
\boldsymbol{L}=\left[\begin{array}{ccc}
\frac{1}{\mid m m_{1}} \frac{\partial x_{1}}{\partial \xi_{1}} & \frac{1}{\mid m_{1}} \frac{\partial x_{2}}{\partial \xi_{1}} & \frac{1}{\mid m_{1}} \frac{\partial x_{3}}{\partial \xi_{1}} \\
n_{2} L_{13}-n_{3} L_{12} & n_{3} L_{11}-n_{1} L_{13} & n_{1} L_{12}-n_{2} L_{11} \\
n_{1} & n_{2} & n_{3}
\end{array}\right],
$$


where $n_{1}, n_{2}$, and $n_{3}$ are the components of unit outward normal. The transformation relationship between global and local stresses is

$$
\sigma_{m n}=L_{r m} L_{s n} \bar{\sigma}_{r s}
$$

Substituting Eqs. (17, 18, A.5-A.10) into Eq. (29), after some lengthy algebra, the boundary stress may be expressed in terms of the displacements and tractions of control points as

$$
\sigma_{m n}=C_{m n j \alpha} u_{j}^{\alpha}+D_{m n j \alpha} t_{j}^{\alpha}
$$

where the coefficients $C_{m n j \alpha}$ and $D_{m n j \alpha}$ are

$$
\begin{aligned}
C_{m n j \alpha}=2 \mu & \left\{\frac { 1 } { 1 - v } \left[L_{1 m} L_{1 n}\left(\frac{\partial \xi_{k}}{\partial \bar{x}_{1}} L_{1 j}+v \frac{\partial \xi_{k}}{\partial \bar{x}_{2}} L_{2 j}\right)\right.\right. \\
+ & \left.L_{2 m} L_{2 n}\left(\frac{\partial \xi_{k}}{\partial \bar{x}_{2}} L_{2 j}+v \frac{\partial \xi_{k}}{\partial \bar{x}_{1}} L_{1 j}\right)\right] \\
+ & \left.\frac{1}{2}\left(L_{1 m} L_{2 n}+L_{2 m} L_{1 n}\right)\left(\frac{\partial \xi_{k}}{\partial \bar{x}_{1}} L_{2 j}+\frac{\partial \xi_{k}}{\partial \bar{x}_{2}} L_{1 j}\right)\right\} \frac{\partial N_{\alpha}}{\partial \xi_{k}} \\
D_{m n j \alpha}= & \left(L_{3 m} L_{1 n}+L_{1 m} L_{3 n}\right) L_{1 j}+\left(L_{2 m} L_{3 n}+L_{3 m} L_{2 n}\right) L_{2 j} \\
& +\left(\frac{v}{1-v} \delta_{m n}+\frac{1-2 v}{1-v} L_{3 m} L_{3 n}\right) L_{3 j},
\end{aligned}
$$

where $N_{\alpha}$ is the basis function associated with the $\alpha$-th control point of the element (refer to Eqs. (17) and (18)), $v$ is Poisson's ratio, $\mu$ is shear modulus, and $\delta_{i j}$ is Kronecker delta.

\subsubsection{Displacement derivative evaluation}

With the help of the local coordinate system in Figure 2, the derivatives of global displacement can be expressed as

$$
u_{m, n}=L_{r m} L_{s n} \bar{u}_{r, s}
$$

When the subscript $j$ does not equal 3, the local derivatives of displacements are

$$
\bar{u}_{i, j}=\frac{\partial \bar{u}_{i}}{\partial \bar{x}_{j}}=\frac{\partial \bar{u}_{i}}{\partial \xi_{k}} \frac{\partial \xi_{k}}{\partial \bar{x}_{j}},
$$

where the subscript $k$ ranges from 1 to 2 . When the subscript $j$ equals to 3 , according to Hooke's law and $\bar{\sigma}_{i 3}=\bar{t}_{i}$, the local derivatives of the displacements 
are

$$
\begin{aligned}
& \frac{\partial \bar{u}_{1}}{\partial \bar{x}_{3}}=\frac{\bar{t}_{1}}{\mu}-\left(\frac{\partial \bar{u}_{3}}{\partial \xi_{k}} \frac{\partial \xi_{k}}{\partial \bar{x}_{1}}\right), \\
& \frac{\partial \bar{u}_{2}}{\partial \bar{x}_{3}}=\frac{\bar{t}_{2}}{\mu}-\left(\frac{\partial \bar{u}_{3}}{\partial \xi_{k}} \frac{\partial \xi_{k}}{\partial \bar{x}_{2}}\right), \\
& \frac{\partial \bar{u}_{3}}{\partial \bar{x}_{3}}=\frac{1-2 v}{\mu(2-2 v)} \bar{t}_{3}-\frac{v}{1-v}\left(\frac{\partial \bar{u}_{1}}{\partial \xi_{k}} \frac{\partial \xi_{k}}{\partial \bar{x}_{1}}+\frac{\partial \bar{u}_{2}}{\partial \xi_{k}} \frac{\partial \xi_{k}}{\partial \bar{x}_{2}}\right),
\end{aligned}
$$

Using Eqs. (17, 18, 27, 33, A.17), the derivatives of displacements are expressed in terms of the displacements and tractions of the control points

$$
u_{m, n}=A_{m n j \alpha} u_{j}^{\alpha}+B_{m n j \alpha} t_{j}^{\alpha},
$$

where the coefficient $A_{m n j \alpha}$ is

$$
\begin{aligned}
A_{m n j \alpha}= & \left(L_{1 m} L_{1 j}+L_{2 m} L_{2 j}+L_{3 m} L_{3 j}\right) L_{1 n} c_{\alpha 1} \\
& +\left(\frac{v}{v-1} L_{3 m}+L_{1 j}-L_{1 m} L_{3 j}\right) L_{3 n} c_{\alpha 1} \\
& +\left(L_{1 m} L_{1 j}+L_{2 m} L_{2 j}+L_{3 m} L_{3 j}\right) L_{2 n} c_{\alpha 2} \\
& +\left(\frac{v}{v-1} L_{3 m}+L_{2 j}-L_{2 m} L_{3 j}\right) L_{3 n} c_{\alpha 2}
\end{aligned}
$$

and coefficients $c_{\alpha 1}$ and $c_{\alpha 2}$ are

$$
\begin{gathered}
c_{\alpha 1}=\frac{\partial \xi_{1}}{\partial \bar{x}_{1}} \frac{\partial N_{\alpha}}{\partial \xi_{1}}+\frac{\partial \xi_{2}}{\partial \bar{x}_{1}} \frac{\partial N_{\alpha}}{\partial \xi_{2}}, \\
c_{\alpha 2}=\frac{\partial \xi_{1}}{\partial \bar{x}_{2}} \frac{\partial N_{\alpha}}{\partial \xi_{1}}+\frac{\partial \xi_{2}}{\partial \bar{x}_{2}} \frac{\partial N_{\alpha}}{\partial \xi_{2}},
\end{gathered}
$$

and the coefficient $B_{m n j \alpha}$ is

$$
B_{m n j \alpha}=\frac{L_{3 n}}{\mu}\left(L_{1 m} L_{1 j}+L_{2 m} L_{2 j}+\frac{1-2 v}{2(1-v)} L_{3 m} L_{3 j}\right) N_{\alpha}
$$

\subsection{Collocation}

The locations of the collocation points (source points) influences the accuracy and stability of the computational results. Different collocation methods are evaluated in reference [27], which concludes that the Greville abscissae produce reasonably accurate and stable results, and therefore they are used here. 
In the nonsingular IGABEM, the source point outward normal needs to be computed (see $n_{k}(\boldsymbol{x})$ in Eq. (24)), which is not uniquely defined if the source point is at the sharp edge or corner. To avoid this difficulty with the outward normals in the multi-patch IGABEM, the Greville abscissae method was modified by moving the edge collocation points inside patches. Two steps are required to implement this collocation method. First, the initial values of the collocation points are chosen as the Greville abscissae in parameter space along each direction of all patches as

$$
\bar{\xi}_{i}=\frac{1}{p}\left(\xi_{i+1}+\xi_{i+2}+\cdots+\xi_{i+p}\right), \quad i=1,2, \ldots, n,
$$

where $p$ is the order of the NURBS basis, $n$ is the number of control points in $\xi$ direction. Then the first and the last collocation points of Eq. (41) are moved inside the patch by

$$
\begin{aligned}
& \bar{\xi}_{1}=\bar{\xi}_{1}+\beta\left(\bar{\xi}_{2}-\bar{\xi}_{1}\right), \\
& \bar{\xi}_{n}=\bar{\xi}_{n}-\beta\left(\bar{\xi}_{n}-\bar{\xi}_{n-1}\right),
\end{aligned}
$$

where $\beta(0<\beta<1)$ is a coefficient that defines how much the collocation points move inside the patch.

An example of comparison of collocation points generated by Greville abscissae method and the improved Greville abscissae method (with coefficient $\beta=0.5$ ) is shown in Figure 3. It is easy to demonstrate that all collocation points are inside the patches and their outward normals are only determined by the patches containing them, and the number of control points is equal to the number of collocation points in each patch.

The number of total collocation points is usually not equal to the number of total control points when the modified Greville abscissae are used because some control points maybe shared by multiple patches but each collocation point only belongs to one patch. This inequality will result in the number of BIEs being greater than the number of unknowns. To solve this problem, the equations for all the collocation points related to a single control point are merged. For example, the index relationship $(i, j, k \Rightarrow m)$ is set up for the three collocation points $\left(C L_{i}, C L_{j}, C L_{k}\right)$ that correspond to the control point $\left(C_{m}\right)$ in Figure 4. Assume that the three BIEs in the initial assembly of Eq. (16) corresponding to the collocation 


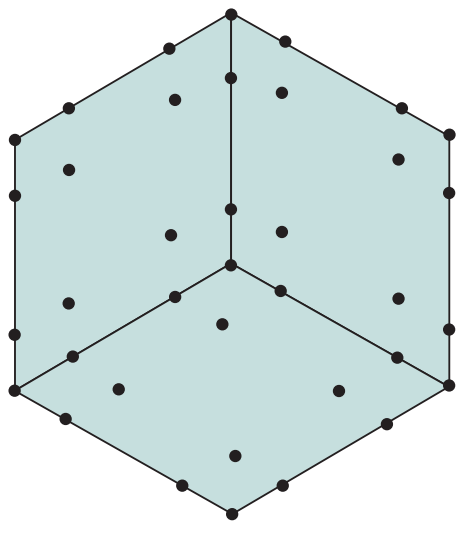

(a)

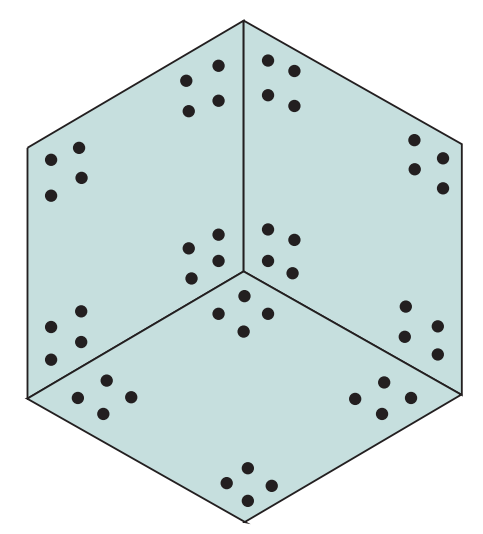

(b)

Figure 3: Two collocation methods: (a) Greville abscissae and (b) improved Greville abscissae. The knot vectors in both parametric direction are $\Xi=[0,0,0,0.5,1,1,1]$.

points with respect to the $\alpha$-th direction are

$$
\begin{aligned}
& A_{\bar{i} 1} q_{1}+A_{\bar{i} 2} q_{2}+\cdots+A_{\bar{i} n} q_{n}=b_{\bar{i}} \\
& A_{\bar{j} 1} q_{1}+A_{\bar{j} 2} q_{2}+\cdots+A_{\bar{j} n} q_{n}=b_{\bar{j}} \\
& A_{\bar{k} 1} q_{1}+A_{\bar{k} 2} q_{2}+\cdots+A_{\bar{k} n} q_{n}=b_{\bar{k}},
\end{aligned}
$$

where $\bar{i}=3 i+\alpha, \bar{j}=3 j+\alpha, \bar{k}=3 k+\alpha$. Using the index relationship $(i, j, k \Rightarrow m)$, the merged BIE for the final equation system Eq. (16) is simply the average

$$
\frac{1}{3}\left(\bar{A}_{\bar{m} 1} q_{1}+\bar{A}_{\bar{m} 2} q_{2}+\cdots+\bar{A}_{\bar{m} n} q_{n}\right)=\bar{b}_{\bar{m}}
$$

in which

$$
\bar{A}_{\bar{m} p}=A_{\bar{i} p}+A_{\bar{j} p}+A_{\bar{k} p}, \quad p=1,2, \ldots, n .
$$

If there are $Q$ collocation points corresponding to a control point, the number of equations in Eq. (43) should be $Q$ and the $\frac{1}{3}$ in Eq. (44) should be replaced with $\frac{1}{Q}$.

\subsection{Implementation}

The final nonsingular system of BIEs are obtained by substituting Eqs. (17, $18,30,36)$ into Eq. (24) to obtain the BIE for the displacements and tractions at 


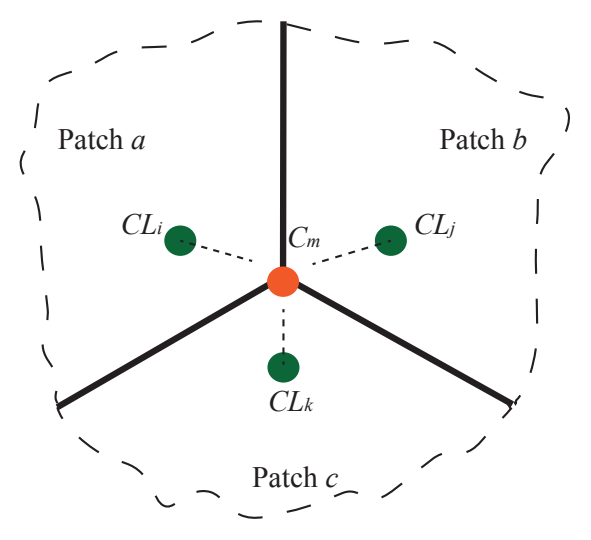

Figure 4: An example of 3 collocation points vs. 1 control point.

the control points

$$
\begin{aligned}
& \int_{\Gamma} T_{l m}(s, \boldsymbol{x}) N_{\alpha}(\boldsymbol{x}) u_{m}^{\alpha}(\boldsymbol{x}) d \Gamma(\boldsymbol{x}) \\
& -N_{\alpha}(s) u_{m}^{\alpha}(s) \int_{\Gamma} T_{l m}(s, \boldsymbol{x}) d \Gamma(\boldsymbol{x}) \\
& -\left(A_{m n j \alpha} u_{j}^{\alpha}(\boldsymbol{s})+B_{m n j \alpha} t_{j}^{\alpha}(s)\right) \int_{\Gamma} T_{l m}(\boldsymbol{s}, \boldsymbol{x}) x(\boldsymbol{x})_{n} d \Gamma(\boldsymbol{x}) \\
& +\left(A_{m n j \alpha} u_{j}^{\alpha}(\boldsymbol{s})+B_{m n j \alpha} t_{j}^{\alpha}(\boldsymbol{s})\right) x(s)_{n} \int_{\Gamma} T_{l m}(s, \boldsymbol{x}) d \Gamma(\boldsymbol{x}) \\
= & \int_{\Gamma} U_{l m}(s, \boldsymbol{x}) N_{\alpha}(\boldsymbol{x}) t_{m}^{\alpha}(\boldsymbol{x}) d \Gamma(\boldsymbol{x}) \\
& -\left(C_{m n j \alpha} u_{j}^{\alpha}(s)+D_{m n j \alpha} t_{j}^{\alpha}(s)\right) \int_{\Gamma} U_{l m}(\boldsymbol{s}, \boldsymbol{x}) n_{n}(\boldsymbol{x}) d \Gamma(\boldsymbol{x}) .
\end{aligned}
$$

Note that the terms taken outside of the integrals simplify the implementation.

In the numerical implementation, the first term of left hand side and right hand side (called field point term for short) in Eq. (46) are computed in each element and then assembled into left hand side or right hand side of the equation system Eq. (16) according to whether $u_{m}^{\alpha}(\boldsymbol{x})$ and $t_{m}^{\alpha}(\boldsymbol{x})$ are unknown or known. Except for the first terms, all other terms (called source point terms for short) consist of a polynomial with respect to the displacements and tractions of the source points (collocation points) and an integral with respect to the field points. 
The integrals are evaluated over each element and summed to get the the integral over the entire boundary $\boldsymbol{\Gamma}$. The integrals are then multiplied by the appropriate polynomial terms and assembled into the equation system according to $u_{j}^{\alpha}(s)$ and $t_{j}^{\alpha}(s)$. Although $\int_{\Gamma} T_{l m}(s, \boldsymbol{x}) d \Gamma(\boldsymbol{x})=-\frac{1}{2} \delta_{l m}$ ( $\boldsymbol{x}$ on a smooth boundary) in terms of the extended first identity in [59], this identity cannot be used in Eq. (46) because the nonsingular BEM needs to use the numerical computational errors of $\int_{\Gamma} T_{l m}(s, x) d \Gamma(x)$ to compensate for the numerical errors of the other integrals. The procedure of the multi-patch nonsingular IGABEM analysis is illustrated in Figure 5.

Nonsingular does not mean that the gradients of the integrands are small or that lower order Gauss integration will accurately evaluate the integrals. The element sub-division method [39] is therefore used to accurately evaluate the integrals when the source and integration points are in the same element.

\section{Example calculations}

The numerical examples were run on a single processor desktop computer with an Intel Core i7 $9603.2 \mathrm{GHz}$ CPU with 12GB of RAM. The operating system was Linux Ubuntu 12.04, and the compiler was gfortran.

\subsection{Unit cube model}

The patch test using a unit cube with 6 patches is from Scott et al. [33]. The boundary conditions are illustrated in Figure 6. Young's modulus E is $10^{4}$, and the Poisson's ratio $v$ is 0.3 . In this model, the analytical solutions of displacement in $x, y$ and $z$ direction of a point $s$ on the cubic surfaces are $0.1 x(s), 0.03 y(s)$ and $0.03 z(s)$.

Meshes of $1 \times 1,2 \times 2,3 \times 3$ and $4 \times 4$ quadratic, cubic and quartic $(p=2, p=3$, and $p=4) 2 D$ NURBS boundary elements for each patch are used in the computations and shown in Figure 7. The knot vector spans are equally spaced. The mean relative error and the maximum relative error (shortly name mean error and max error below) of the node displacement components are given in Table 1. When a point is shared by several surfaces, only the common displacement components of those surfaces in Table 1 are compared.

\subsubsection{Influence of shift coefficient $\beta$}

We chose different $\beta$ values in Eq.(42) from 0.1 to 0.7 to analyze the influences of the coefficient $\beta$ in the nonsingular IGABEM. A $8 \times 8$ Gauss quadrature rule is 


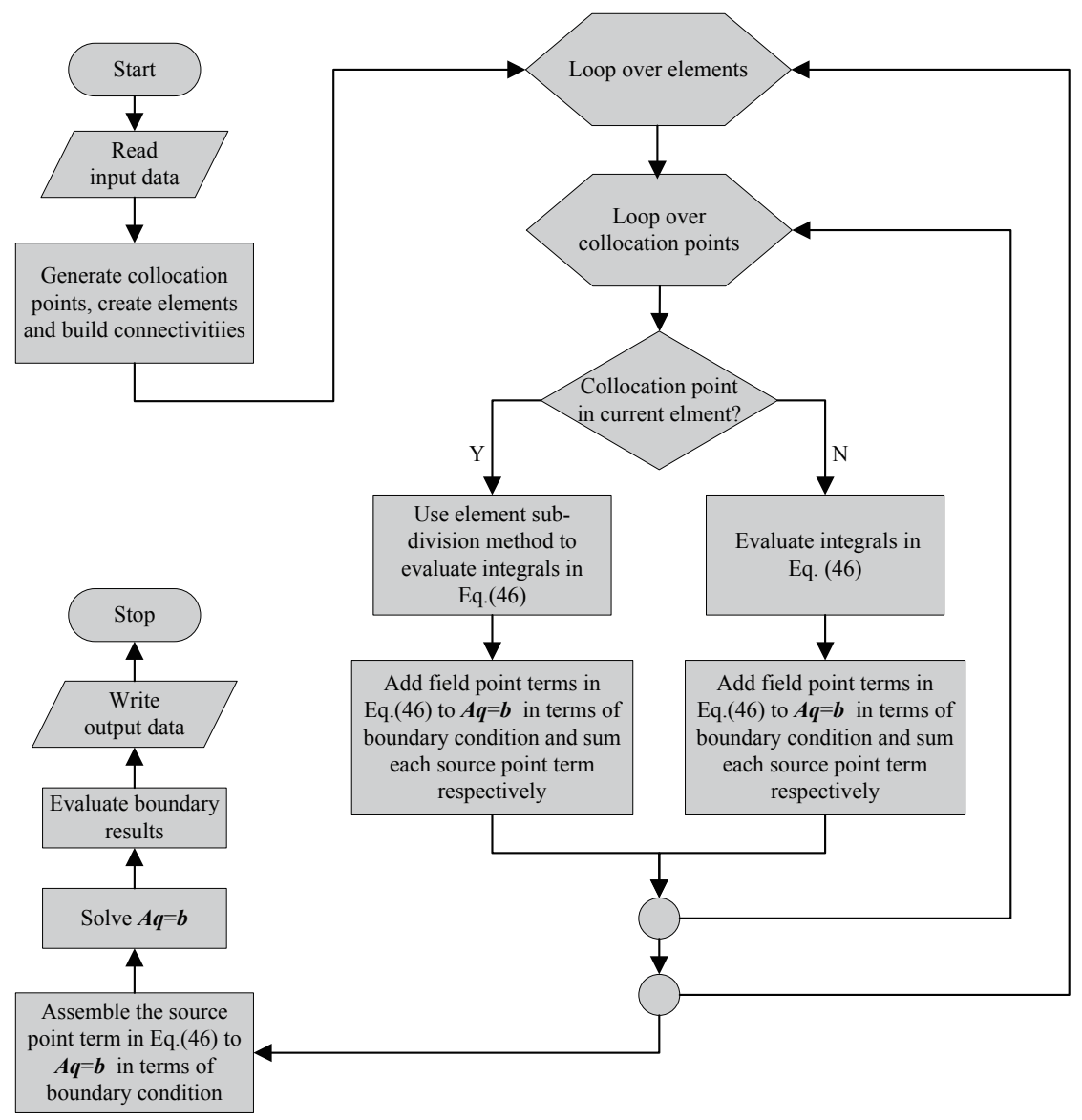

Figure 5: Flowchart of the multi-patch nonsingular IGABEM analysis.

used in Mesh 2 and Mesh 4 with quadratic, cubic and quartic NURBS elements. The errors are shown in Figure 8.

In Figure 8 (a), the minimal mean errors of Mesh 2 and Mesh 4 are at $\beta=0.6$ and $\beta=0.7$ respectively, and both of the minimal max errors are at $\beta=0.5$. In Figure 8 (b), the minimal mean errors of Mesh 2 and Mesh 4 are at $\beta=0.6$ and $\beta=0.5$ respectively, and both of the minimal max errors are at $\beta=0.5$. In Figure 8 (c), the minimal mean errors of both Mesh 2 and Mesh 4 and the max error of Mesh 4 are at $\beta=0.5$. Based on these results, the shift coefficient $\beta=$ 0.5 performs best for the different element orders and it is used in the following examples in this paper.

The displacement results for Mesh 4 with $p=2$ are shown in Figure 9. The 


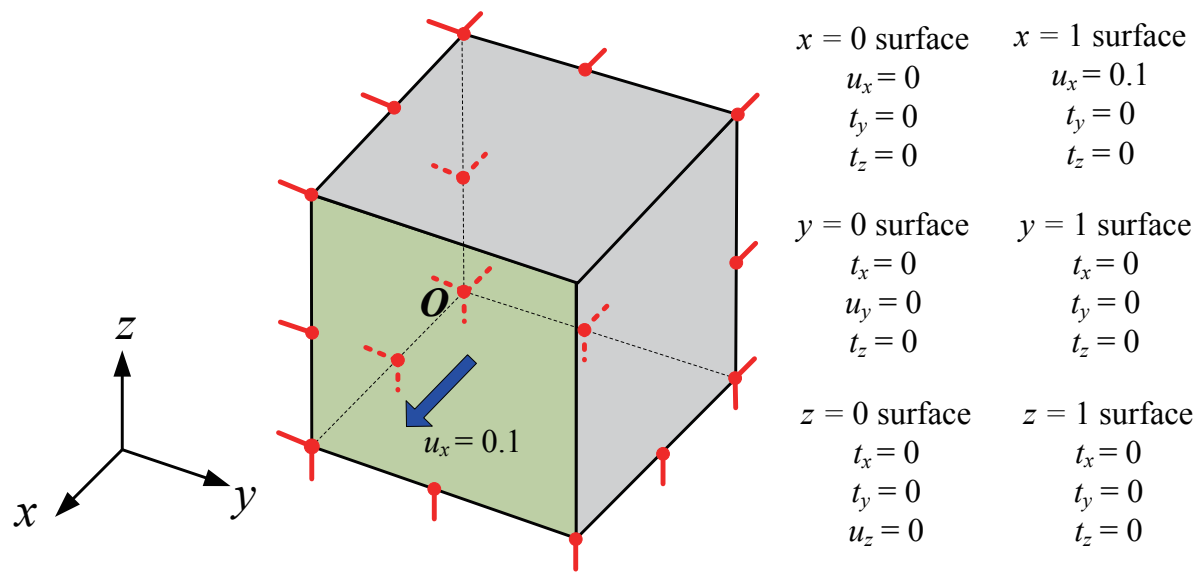

Figure 6: A patch test cube model.

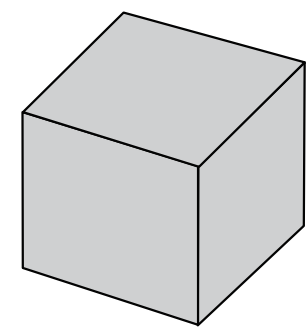

(a) Mesh 1

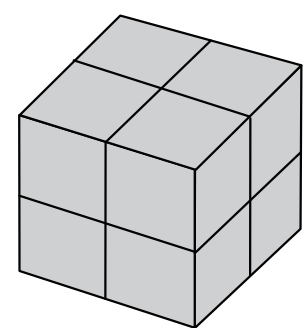

(b) Mesh 2

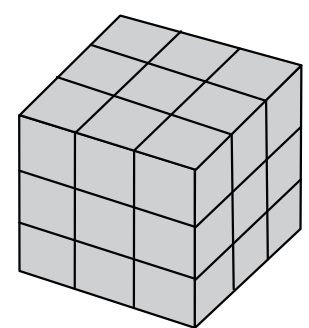

(c) Mesh 3

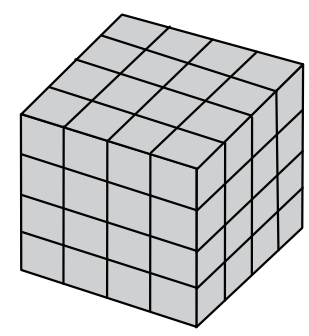

(d) Mesh 4

Figure 7: Cube model meshes.

mean error and the max error between the nonsingular IGABEM results and the analytical solutions are $1.3534 \times 10^{-5}$ and $2.4489 \times 10^{-4}$. Note that their percent errors are approximately $0.001 \%$ and $0.02 \%$ respectively, demonstrating the high accuracy of the nonsingular IGABEM.

\subsubsection{Influence of the number Gauss points}

The mean errors of Mesh 1 to Mesh 4 with number of Gauss points varied from 5 to 8 in each direction of each element are shown in Figure 10. The numerical solutions converge to the analytical solutions as the number of quadrature points increase, and $p=3$ and $p=4$ converge faster than $p=2$ as expected. This illustrates the sensitivity of the BEM accuracy to the quadrature in comparison to FEM, where the accuracy rarely improves (and may actually decay) once a "sufficient" number of integration points are used. 
Table 1: Compared displacement components of different surfaces.

\begin{tabular}{cccccc}
$\mathrm{X}=0$ & $\mathrm{x}=1$ & $\mathrm{y}=0$ & $\mathrm{y}=1$ & $\mathrm{z}=0$ & $\mathrm{z}=1$ \\
surface & surface & surface & surface & surface & surface \\
\hline & & $u_{x}$ & $u_{x}$ & $u_{x}$ & $u_{x}$ \\
$u_{y}$ & $u_{y}$ & & $u_{y}$ & $u_{y}$ & $u_{y}$ \\
$u_{z}$ & $u_{z}$ & $u_{z}$ & $u_{z}$ & & $u_{z}$ \\
\hline
\end{tabular}

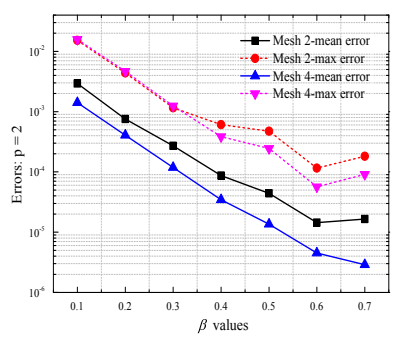

(a) $p=2$

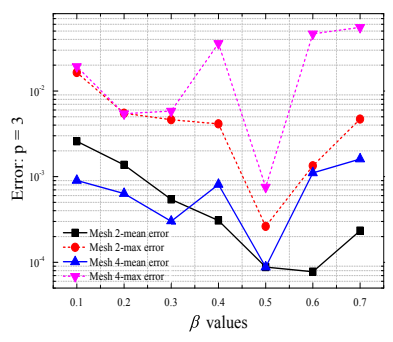

(b) $p=3$

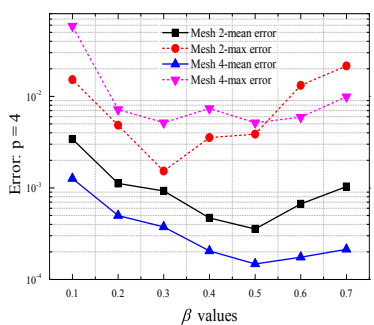

(c) $p=4$

Figure 8: Errors of different $\beta$ values.

\subsubsection{Influence of element sub-division}

As mentioned previously, when the source and integration points are in the same element, the gradients in the $1 / r$ and $1 / r^{2}$ terms may be very high and the standard Gauss quadrature may not provide adequate accuracy. Two simple element sub-division schemes can be used to address this problem.

The mean errors of Mesh 4 using $8 \times 8$ Gauss quadrature rule are shown in Table 2. The triangular sub-division scheme achieves higher accuracy than the rectangular sub-division one because the triangular sub-division concentrates the

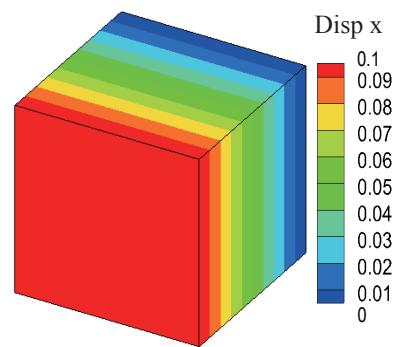

(a) Displacement in $x$

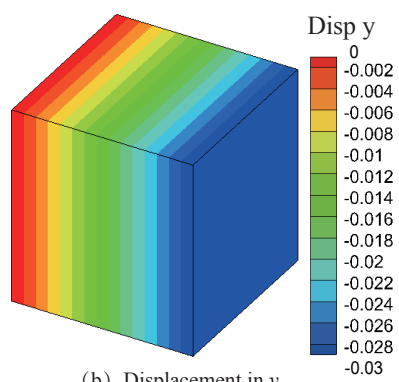

(b) Displacement in $y$

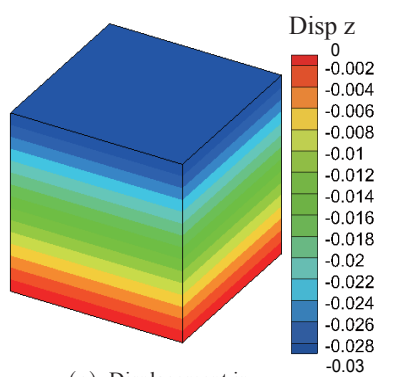

(c) Displacement in $z$

Figure 9: Displacment results of the cube. 


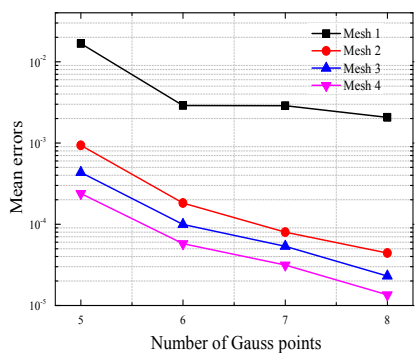

(a) $p=2$

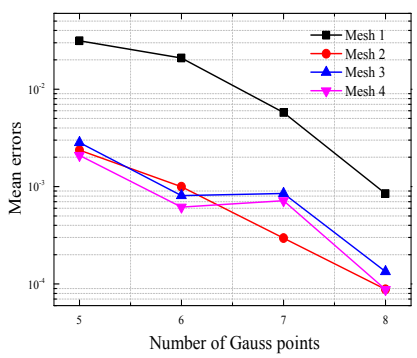

(a) $p=3$

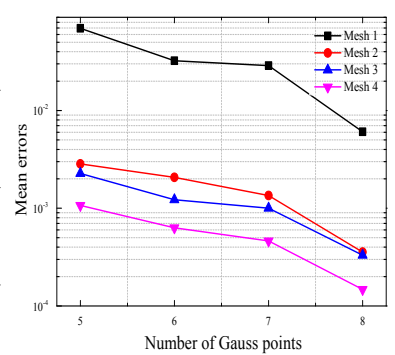

(a) $p=4$

Figure 10: Errors of different Gauss points.
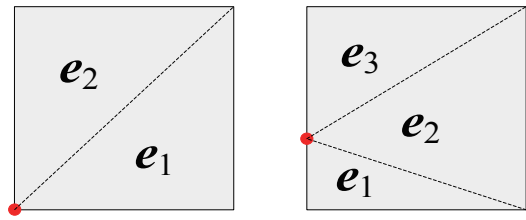

(a)
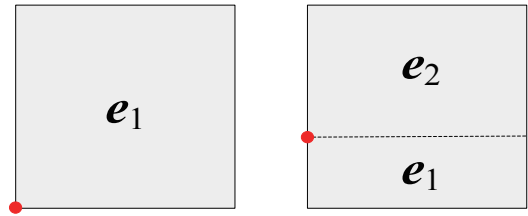

(b)
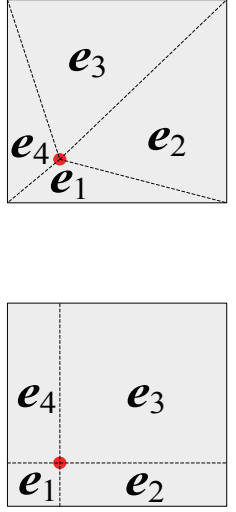

\section{(b)}

Figure 11: Element sub-division schemes: (a) triangular sub-division and (b)rectangular subdivision.

integration points near the source point. With superior accuracy, the triangular sub-division scheme is used in this study.

\subsubsection{Nonsingular IGABEM versus IGABEM}

Mesh 4 is used to compare the nonsingular IGABEM (NSIGABEM) to the conventional IGABEM. In the conventional IGABEM, the element sub-division technique employed by Lachat and Watson [39] is used for the weakly singular integrals, and the weakly singular form of the $T$ kernel in Eq. (20) with the subdivision technique is used to complete the strongly singular integrals.

The mean errors of the NSIGABEM and the IGABEM with number of Gauss points from 5 to 8 are shown in Figure 12. The accuracy of the NSIGABEM is about one digit higher than that of the IGABEM. This demonstrates the high 
Table 2: Mean errors of the element sub-division schemes.

\begin{tabular}{cccc}
\hline sub-division & $p=2$ & $p=3$ & $p=4$ \\
\hline triangle & $1.352 \times 10^{-5}$ & $8.778 \times 10^{-5}$ & $1.479 \times 10^{-4}$ \\
rectangle & $8.909 \times 10^{-5}$ & $1.726 \times 10^{-4}$ & $2.428 \times 10^{-4}$ \\
\hline
\end{tabular}
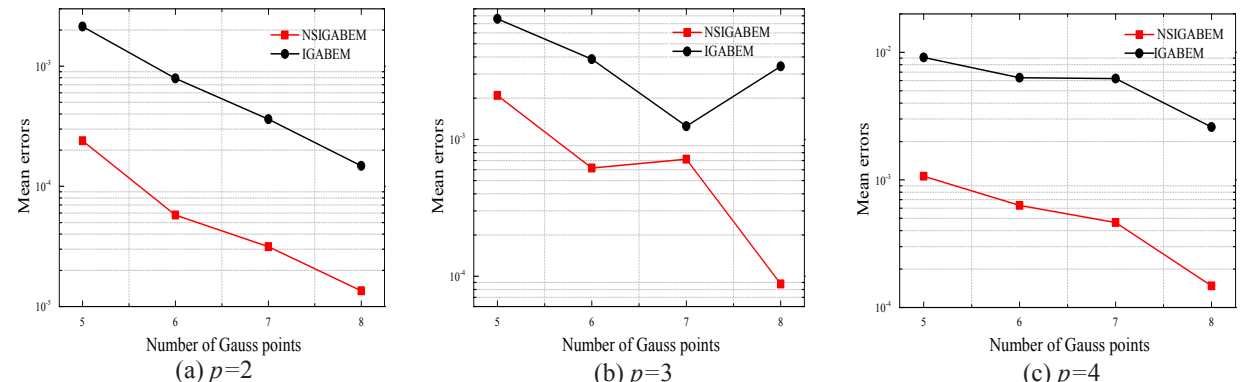

Figure 12: NSIGABEM.vs.IGABEM: Error.

accuracy of the NSIGABEM.

In order to compare the CPU time fairly, only the integration computation part is compared because all the other costs are the same between the NSIGABEM and the IGABEM. The CPU time is shown in Figure 13. The time cost ratio of the integration is shown in Table 3. The CPU time ratio is just a little bigger than 1 , which means that the efficiency of the NSIGABEM only little lower than that of the IGABEM in integration. Furthermore, the integration takes approximately $1 / 2$ of the total BEM computation in general, so the extra cost of the integration of the NSIGABEM is comparatively small. The CPU time ratio decreases as the element order increases because the number of common terms evaluated in the NSIGABEM and IGABEM formulations increases with $p$ in the integral evaluation.

Table 3: Time cost ratio: NSIGABEM/IGABEM.

\begin{tabular}{cccc}
\hline$N_{g p}$ & $p=2$ & $p=3$ & $p=4$ \\
\hline 5 & 1.395 & 1.246 & 1.094 \\
6 & 1.450 & 1.289 & 1.157 \\
7 & 1.515 & 1.316 & 1.200 \\
8 & 1.550 & 1.318 & 1.201 \\
\hline
\end{tabular}




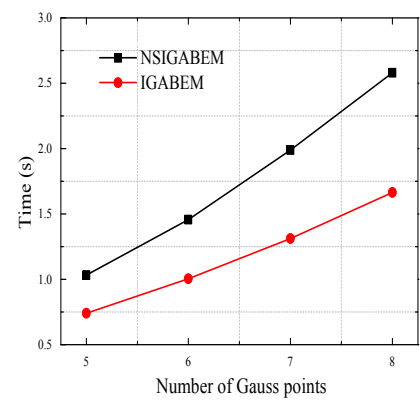

(a) $p=2$

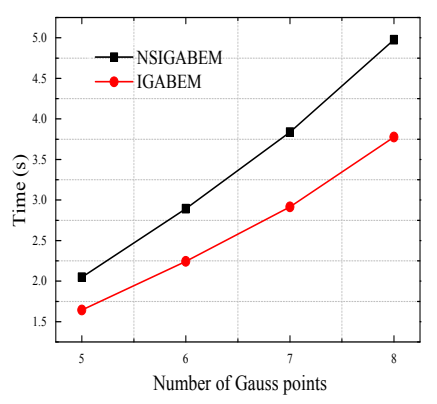

(b) $p=3$

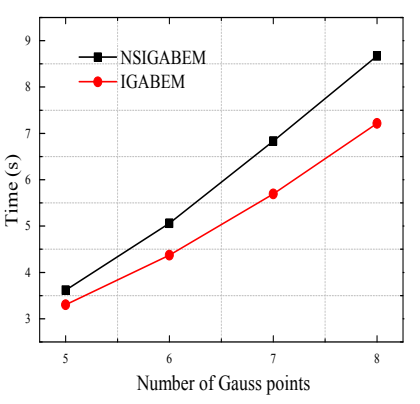

(c) $p=4$

Figure 13: NSIGABEM.vs.IGABEM: Time cost of integration part.

\subsection{Solid circular cylinder model}

A quarter of a solid circular cylinder is subjected to a perpendicular tension loading as shown in Figure 14. The exact solution of displacement in $z$ direction for this case is

$$
u_{z}(s)=T z(s) / E,
$$

where $s$ is a point belonging to the circular cylinder.

The coarse mesh (Figure 15), similar to the circular cylinder model in Reference [33], has 24 NURBS elements distributed over 6 patches. A $20 \times 20$ Gauss quadrature rule is used to integrate each element. Table 4 summarizes the results where only the points on the unconstrained surfaces are used in the error evaluation of displacement in $z$ direction.

The mean errors and the max errors of the NSIGABEM are smaller than that of the IGABEM which demonstrates the high accuracy of the NSIGABEM. In Reference [33], the maximum error in radial displacment of the circular cylinder subjected to internal pressure loading is about $1.116 \times 10^{-3}$, where a cubic element and a $30 \times 30$ Gauss quadrature rule is used. From Table 4, the max error of the NSIGABEM with cubic NURBS element in the press-loading direction is only $5.550 \times 10^{-4}$ which is approximate half of that in [33], and the number of Gauss points used in each element is less than half of that in [33].

\subsection{Arch model}

The last example is an arch model whose bottom surfaces are fixed and the top surface is subjected uniform pressure as shown in Figure 16. The entire geometry consists of 18 patches. The mesh contains 72 quadratic NURBS elements as shown in Figure 17. The magnitude of the displacement of the NSIGABEM so- 


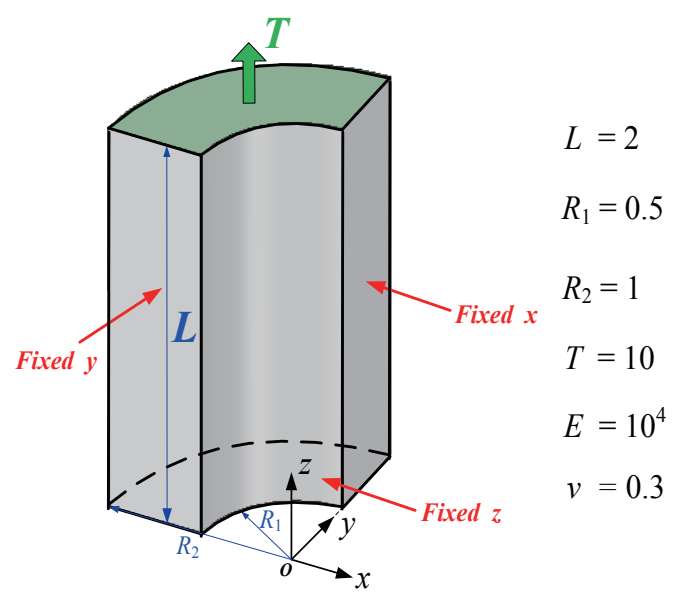

Figure 14: Circular cylinder model.

Table 4: Results of circular cylinder model.

\begin{tabular}{ccccccc}
\hline$p$ & & NSIGABEM & & & IGABEM & \\
& mean error & max error & integ-time $(\mathrm{s})$ & mean error & max error & integ-time (s) \\
\hline 2 & $1.871 \times 10^{-4}$ & $7.000 \times 10^{-4}$ & 2.064 & $4.151 \times 10^{-4}$ & $1.340 \times 10^{-3}$ & 1.304 \\
3 & $1.011 \times 10^{-4}$ & $5.550 \times 10^{-4}$ & 4.708 & $2.249 \times 10^{-3}$ & $8.785 \times 10^{-3}$ & 3.576 \\
4 & $2.319 \times 10^{-4}$ & $3.900 \times 10^{-4}$ & 9.021 & $1.746 \times 10^{-4}$ & $6.150 \times 10^{-3}$ & 7.256 \\
\hline
\end{tabular}

lution is plotted in Figure 18 (a), and an ANSYS reference solution using 16384 hexahedral elements is shown in Figure 18 (b). The overall displacment distribution of NSIGABEM is the same as that of ANSYS and the relative error at the maximum displacement location is about $3 \%$ demonstrating the coarse mesh accuracy of the NSIGAEBEM.

\section{Conclusions}

Traditional formulations of BEM generate integrals that are singular or weakly singular, and complicated, specialized integration methods are used to evaluate them numerically. Many of the methods are restricted to simple basis functions such as linear triangles or bilinear quadrilaterals. The recent drive towards introducing isogeometric analysis concepts into BEM has challenged these integration methods, and the expense associated with the increased number of integration points required for accurate solutions reduces the advantages of the higher order accuracy obtained by using basis functions from CAD. 


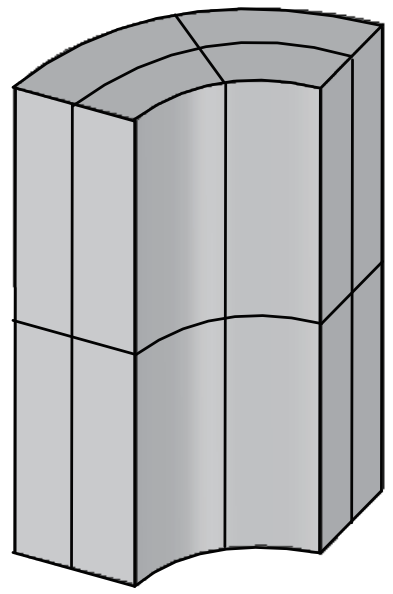

Figure 15: Circular cylinder mesh.

Many nonsingular BEM have been proposed, but they have not replaced the traditional formulations for lower order elements. This is most likely due to the comparative complexity of the integrals in their formulation compared to the standard formulations. This paper has restructured the integrals, moving some terms outside of the integrals to simplify their evaluation and to improve the efficiency of the calculation. The derivatives required for the nonsingular formulation that do not appear in the standard formulations were developed in terms of the solution variables.

A modified set of Greville abscissae with the first and last points moved to the element interiors was found to provide an accurate set of collocation points. A simple averaging technique was demonstrated to be effective for merging the surplus collocations points along edges and corners. Test calculations show that the best value of the shift coefficient $\beta$ is $1 / 2$.

Numerical examples have demonstrated that the nonsingular method is at least as accurate as traditional methods of equal order, and in some cases, may be more accurate. Compared to the traditional formulation, the nonsingular formulation appears to be only slightly more expensive overall per collocation point.

The greatest benefit of the nonsingular IGABEM is that it can be used with irregular trimmed NURBS $[65,66]$ where applying conventional methods for addressing the singularities are much more difficult than for regular triangular and quadrilateral elements. The present formulation is being combined with an optimal integration method for trimmed NURBS [67] is currently being developed and will be presented in a future paper. 


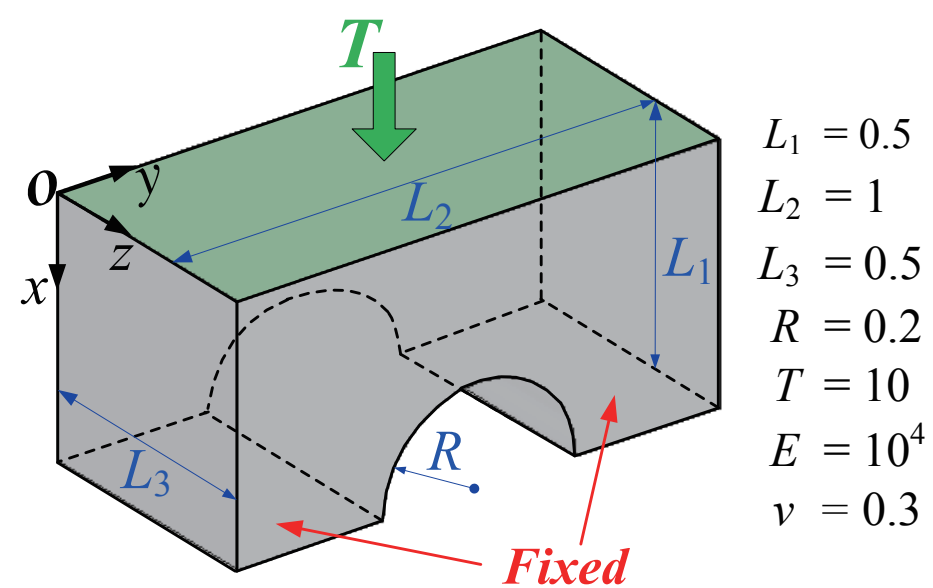

Figure 16: Arch model.

\section{Acknowledgments}

The support for this research and Y. J. Wang by NSF grant CMMI-1068106 is gratefully acknowledged.

\section{Appendix A. Expressions for the local stresses and displacement derivatives in terms of global equivalents}

The local stresses and displacement derivatives are expressed in terms of global variables according to Eqs. (29) and (33). Their derivatives, required by the nonsingular formulation, are also required, and we present their derivation in detail here.

The local tangential strains may be expressed in terms of the derivatives of the displacements as

$$
\bar{\varepsilon}_{i j}=\frac{1}{2}\left(\frac{\partial \bar{u}_{i}}{\partial \bar{x}_{j}}+\frac{\partial \bar{u}_{j}}{\partial \bar{x}_{i}}\right) .
$$

Using Hooke's law and eliminating the local normal strain $\bar{\sigma}_{33}$, we obtain

$$
\begin{gathered}
\bar{\sigma}_{11}=\frac{2 \mu}{1-v}\left(\bar{\varepsilon}_{11}+v \bar{\varepsilon}_{22}\right)+\frac{v}{1-v} \bar{\sigma}_{33}, \\
\bar{\sigma}_{22}=\frac{2 \mu}{1-v}\left(\bar{\varepsilon}_{22}+v \bar{\varepsilon}_{11}\right)+\frac{v}{1-v} \bar{\sigma}_{33}, \\
\bar{\sigma}_{12}=2 \mu \bar{\varepsilon}_{12},
\end{gathered}
$$




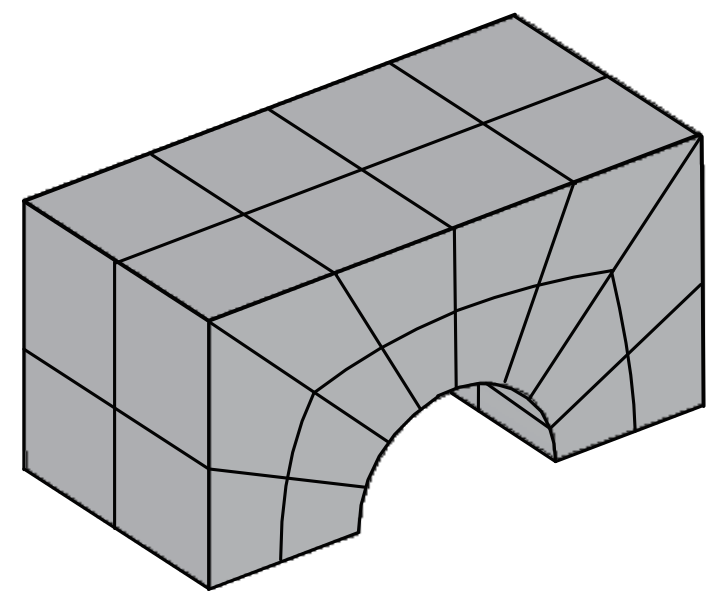

Figure 17: Arch mesh.

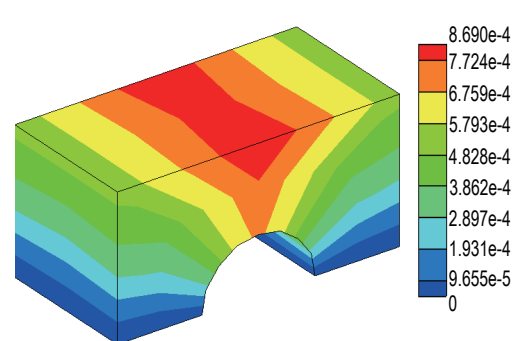

(a) NSIGABEM

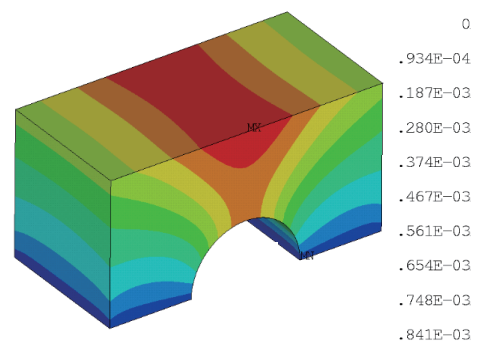

(b) ANSYS

Figure 18: Arch displacement result.

where $\mu$ and $v$ are shear modulus and Poisson's ratio, respectively, and the equilibrium requires that

$$
\begin{aligned}
& \bar{\sigma}_{13}=\bar{t}_{1}=L_{1 j} t_{j}, \\
& \bar{\sigma}_{23}=\bar{t}_{2}=L_{2 j} t_{j}, \\
& \bar{\sigma}_{33}=\bar{t}_{3}=L_{3 j} t_{j},
\end{aligned}
$$

where $L_{i j}$ are defined in Eq. (28).

Taking these results and Eqs. (27) and (34) together, the local stresses in Eqs. (A.2-A.4) may be expressed in terms of global Cartesian variables as

$$
\bar{\sigma}_{11}=\frac{2 \mu}{1-v}\left(\frac{\partial \xi_{k}}{\partial \bar{x}_{1}} L_{1 j}+v \frac{\partial \xi_{k}}{\partial \bar{x}_{2}} L_{2 j}\right) \frac{\partial u_{j}}{\partial \xi_{k}}+\frac{v}{1-v} L_{3 j} t_{j},
$$




$$
\begin{gathered}
\bar{\sigma}_{22}=\frac{2 \mu}{1-v}\left(\frac{\partial \xi_{k}}{\partial \bar{x}_{2}} L_{2 j}+v \frac{\partial \xi_{k}}{\partial \bar{x}_{1}} L_{1 j}\right) \frac{\partial u_{j}}{\partial \xi_{k}}+\frac{v}{1-v} L_{3 j} t_{j}, \\
\bar{\sigma}_{12}=\mu\left(\frac{\partial \xi_{k}}{\partial \bar{x}_{2}} L_{1 j}+\frac{\partial \xi_{k}}{\partial \bar{x}_{1}} L_{2 j}\right) \frac{\partial u_{j}}{\partial \xi_{k}}
\end{gathered}
$$

Now the expressions of the local displacement derivatives are derived as follows. When the subscript $j$ equals 1 or 2, substituting Eq. (27) to Eq. (34) gives

$$
\bar{u}_{i, j}=L_{i j} \frac{\partial u_{j}}{\partial \xi_{k}} \frac{\partial \xi_{k}}{\partial \bar{x}_{j}} .
$$

When subscript $j$ equals 3 , the expressions of the local displacement derivatives are a little complicated and therefore the full derivation is given here. We first recall the the stress-strain relation

$$
\sigma_{i j}=\lambda \varepsilon_{k k} \delta_{i j}+2 \mu \varepsilon_{i j},
$$

where $\lambda$ and $\mu$ are the Lamé constants and

$$
\lambda=\frac{2 \mu \nu}{1-2 v} \text {. }
$$

According to Eqs. (A.1), (A.12) and (A.13), the local stresses $\bar{\sigma}_{11}, \bar{\sigma}_{22}$ and $\bar{\sigma}_{22}$ may be expressed as

$$
\begin{gathered}
\bar{\sigma}_{13}=\mu\left(\frac{\partial \bar{u}_{1}}{\partial \bar{x}_{3}}+\frac{\partial \bar{u}_{3}}{\partial \bar{x}_{1}}\right), \\
\bar{\sigma}_{23}=\mu\left(\frac{\partial \bar{u}_{2}}{\partial \bar{x}_{3}}+\frac{\partial \bar{u}_{3}}{\partial \bar{x}_{2}}\right), \\
\bar{\sigma}_{33}=\frac{2 \mu \nu}{1-2 v}\left(\frac{\partial \bar{u}_{1}}{\partial \bar{x}_{1}}+\frac{\partial \bar{u}_{2}}{\partial \bar{x}_{2}}\right)+2 \mu\left(\frac{\partial \bar{u}_{3}}{\partial \bar{x}_{3}}\right),
\end{gathered}
$$

Using $\bar{\sigma}_{i 3}=\bar{t}_{i}$ and Eq. (34) to replace the corresponding variables in Eqs. (A.14A.16), Eq. (35) is obtained. Substituting Eq. (27) to Eq. (35) gives the local displacement derivatives in terms of global variables as

$$
\begin{aligned}
& \frac{\partial \bar{u}_{1}}{\partial \bar{x}_{3}}=\frac{L_{1 j} t_{j}}{\mu}-\left(L_{3 j} \frac{\partial u_{j}}{\partial \xi_{k}} \frac{\partial \xi_{k}}{\partial \bar{x}_{1}}\right) \\
& \frac{\partial \bar{u}_{2}}{\partial \bar{x}_{3}}=\frac{L_{2 j} t_{j}}{\mu}-\left(L_{3 j} \frac{\partial u_{j}}{\partial \xi_{k}} \frac{\partial \xi_{k}}{\partial \bar{x}_{2}}\right) \\
& \frac{\partial \bar{u}_{3}}{\partial \bar{x}_{3}}=\frac{1-2 v}{\mu(2-2 v)} L_{3 j} t_{j}-\frac{v}{1-v}\left(L_{1 j} \frac{\partial u_{j}}{\partial \xi_{k}} \frac{\partial \xi_{k}}{\partial \bar{x}_{1}}+L_{2 j} \frac{\partial u_{j}}{\partial \xi_{k}} \frac{\partial \xi_{k}}{\partial \bar{x}_{2}}\right)
\end{aligned}
$$




\section{References}

[1] T. J. R. Hughes, J. A. Cottrell, and Y. Bazilevs. Isogeometric analysis: CAD, finite elements, NURBS, exact geometry, and mesh refinement. Computer Methods in Applied Mechanics and Engineering, 194:4135-4195, 2005.

[2] J. A. Cottrell, T. J. R. Hughes, and Y. Bazilevs. Isogeometric Analysis: Toward Integration of CAD and FEA. Wiley, Chichester, 2009.

[3] J.A. Cottrell, A. Reali, Y. Bazilevs, and T.J.R. Hughes. Isogeometric analysis of structural vibrations. Computer Methods in Applied Mechanics and Engineering, 195(41):5257-5296, 2006.

[4] Y. Bazilevs, C. Michler, V.M. Calo, and T.J.R. Hughes. Isogeometric variational multiscale modeling of wall-bounded turbulent flows with weakly enforced boundary conditions on unstretched meshes. Computer Methods in Applied Mechanics and Engineering, 199(13):780-790, 2010.

[5] I. Akkerman, Y. Bazilevs, C.E. Kees, and M.W. Farthing. Isogeometric analysis of free-surface flow. Journal of Computational Physics, 230(11):41374152, 2011.

[6] M.C. Hsu and Y. Bazilevs. Fluid-structure interaction modeling of wind turbines: simulating the full machine. Computational Mechanics, 50(6):821833, 2012.

[7] Y. Bazilevs, M.C Hsu, and M.A. Scott. Isogeometric fluid-structure interaction analysis with emphasis on non-matching discretizations, and with application to wind turbines. Computer Methods in Applied Mechanics and Engineering, 249:28-41, 2012.

[8] Y. Bazilevs, K. Takizawa, T.E. Tezduyar, M.C. Hsu, N. Kostov, and S. McIntyre. Aerodynamic and FSI analysis of wind turbines with the ALE-VMS and ST-VMS methods. Archives of Computational Methods in Engineering, pages $1-40,2014$.

[9] S.B. Raknes, X. Deng, Y. Bazilevs, D.J. Benson, K.M. Mathisen, and T. Kvamsdal. Isogeometric rotation-free bending-stabilized cables: Statics, dynamics, bending strips and coupling with shells. Computer Methods in Applied Mechanics and Engineering, 263:127-143, 2013. 
[10] D. J. Benson, Y. Bazilevs, M.-C. Hsu, and T. J. R. Hughes. Isogeometric shell analysis: The Reissner-Mindlin shell. Computer Methods in Applied Mechanics and Engineering, 199:276-289, 2010.

[11] D. J. Benson, Y. Bazilevs, M.-C. Hsu, and T. J. R. Hughes. A large deformation, rotation-free, isogeometric shell. Computer Methods in Applied Mechanics and Engineering, 200:1367-1378, 2011.

[12] D.J. Benson, S. Hartmann, Y. Bazilevs, M.-C. Hsu, and T.J.R. Hughes. Blended isogeometric shells. Computer Methods in Applied Mechanics and Engineering, 255:133-146, 2013.

[13] L. De Lorenzis, I. Temizer, P. Wriggers, and G. Zavarise. A large deformation frictional contact formulation using NURBS-based isogeometric analysis. International Journal for Numerical Methods in Engineering, 87:12781300, 2011.

[14] I. Temizer, P. Wriggers, and T.J.R. Hughes. Three-dimensional mortar-based frictional contact treatment in isogeometric analysis with NURBS. Computer Methods in Applied Mechanics and Engineering, 223-224:173-185, 2012.

[15] E. De Luycker, D. J. Benson, T. Belytschko, Y. Bazilevs, and M.-C. Hsu. XFEM in isogeometric analysis for linear fracture mechanics. International Journal for Numerical Methods in Engineering, 87:541-565, 2011.

[16] X.P. Qian. Full analytical sensitivities in NURBS based isogeometric shape optimization. Computer Methods in Applied Mechanics and Engineering, 199(29):2059-2071, 2010.

[17] H. Azegami, S. Fukumoto, and T. Aoyama. Shape optimization of continua using NURBS as basis functions. Structural and Multidisciplinary Optimization, 47(2):247-258, 2013.

[18] Y.D. Seo, H.J. Kim, and S.K. Youn. Isogeometric topology optimization using trimmed spline surfaces. Computer Methods in Applied Mechanics and Engineering, 199(49):3270-3296, 2010.

[19] L. Dedè, M.J. Borden, and T.J.R. Hughes. Isogeometric analysis for topology optimization with a phase field model. Archives of Computational Methods in Engineering, 19(3):427-465, 2012. 
[20] Y. Bazilevs, V.M. Calo, J.A. Cottrell, J.A. Evans, T.J.R. Hughes, S. Lipton, M.A. Scott, and T.W. Sederberg. Isogeometric analysis using T-splines. Computer Methods in Applied Mechanics and Engineering, 199(5):229263, 2010.

[21] M. A. Scott, X. Li, T. W. Sederberg, and T. J. R. Hughes. Local refinement of analysis-suitable T-splines. Computer Methods in Applied Mechanics and Engineering, 2012. Accepted for publication.

[22] N. Nguyen-Thanh, J. Kiendl, H. Nguyen-Xuan, R. Wüchner, K.U. Bletzinger, Y. Bazilevs, and T. Rabczuk. Rotation free isogeometric thin shell analysis using PHT-splines. Computer Methods in Applied Mechanics and Engineering, 200(47):3410-3424, 2011.

[23] A.-V. Vuong, C. Giannelli, B. Jüttler, and B. Simeon. A hierarchical approach to adaptive local refinement in isogeometric analysis. Computer Methods in Applied Mechanics and Engineering, 200(49):3554-3567, 2011.

[24] D. Schillinger, L. Dede, M.A. Scott, J.A. Evans, M.J. Borden, E. Rank, and T.J.R. Hughes. An isogeometric design-through-analysis methodology based on adaptive hierarchical refinement of NURBS, immersed boundary methods, and T-spline CAD surfaces. Computer Methods in Applied Mechanics and Engineering, 249:116-150, 2012.

[25] H.Speleers, C. Manni, F. Pelosi, and M.L. Sampoli. Isogeometric analysis with Powell-Sabin splines for advection-diffusion-reaction problems. Computer Methods in Applied Mechanics and Engineering, 221:132-148, 2012.

[26] H.Speleers, C. Manni, and F. Pelosi. From NURBS to NURPS geometries. Computer Methods in Applied Mechanics and Engineering, 255:238-254, 2013.

[27] K. Li and X.P. Qian. Isogeometric analysis and shape optimization via boundary integral. Computer-Aided Design, 43(11):1427-1437, 2011.

[28] J.L. Gu, J.M. Zhang, and G.Y. Li. Isogeometric analysis in bie for 3-d potential problem. Engineering Analysis with Boundary Elements, 36(5):858$865,2012$.

[29] P.K. Banerjee and R. Butterfield. The boundary element method for engineers, volume 17. London:Pentech Press, 1978. 
[30] P.K. Banerjee and R. Butterfield. Boundary element methods in engineering science, volume 17. McGraw-Hill London, 1981.

[31] T. Takahashi and T.Matsumoto. An application of fast multipole method to isogeometric boundary element method for laplace equation in two dimensions. Engineering Analysis with Boundary Elements, 36(12):1766-1775, 2012.

[32] R.N. Simpson, S.P.A. Bordas, J. Trevelyan, and T. Rabczuk. A twodimensional isogeometric boundary element method for elastostatic analysis. Computer Methods in Applied Mechanics and Engineering, 209:87-100, 2012.

[33] M.A. Scott, R.N. Simpson, J.A. Evans, S. Lipton, S.P.A. Bordas, T.J.R. Hughes, and T.W. Sederberg. Isogeometric boundary element analysis using unstructured T-splines. Computer Methods in Applied Mechanics and Engineering, 254:197-221, 2013.

[34] C. Politis, A.I. Ginnis, P.D. Kaklis, K. Belibassakis, and C. Feurer. An isogeometric bem for exterior potential-flow problems in the plane. In 2009 SIAM/ACM Joint Conference on Geometric and Physical Modeling, pages 349-354. ACM, 2009.

[35] M.J. Peake, J. Trevelyan, and G. Coates. Extended isogeometric boundary element method (XIBEM) for two-dimensional Helmholtz problems. Computer Methods in Applied Mechanics and Engineering, 259:93-102, 2013.

[36] A.I. Ginnis, K.V. Kostas, C.G. Politis, P.D. Kaklis, K.A. Belibassakis, Th.P. Gerostathis, M.A. Scott, and T.J.R. Hughes. Isogeometric boundary-element analysis for the wave-resistance problem using T-splines. Computer Methods in Applied Mechanics and Engineering, 2014.

[37] M. Guiggiani and A. Gigante. A general algorithm for multidimensional cauchy principal value integrals in the boundary element method. Journal of Applied Mechanics, 57(4):906-915, 1990.

[38] M. Guiggiani, G. Krishnasamy, T.J. Rudolphi, and F.J. Rizzo. A general algorithm for the numerical solution of hypersingular boundary integral equations. Journal of applied mechanics, 59(3):604-614, 1992. 
[39] J.C. Lachat and J.O. Watson. Effective numerical treatment of boundary integral equations: A formulation for three-dimensional elastostatics. International Journal for Numerical Methods in Engineering, 10(5):991-1005, 1976.

[40] X.W. Gao. The radial integration method for evaluation of domain integrals with boundary-only discretization. Engineering Analysis with Boundary Elements, 26(10):905-916, 2002.

[41] X.W. Gao. An effective method for numerical evaluation of general 2D and 3D high order singular boundary integrals. Computer methods in applied mechanics and engineering, 199(45):2856-2864, 2010.

[42] J.C.F. Telles. A self-adaptive co-ordinate transformation for efficient numerical evaluation of general boundary element integrals. International Journal for Numerical Methods in Engineering, 24(5):959-973, 1987.

[43] J.C.F. Telles and R.F. Oliveira. Third degree polynomial transformation for boundary element integrals: further improvements. Engineering Analysis with Boundary Elements, 13(2):135-141, 1994.

[44] B.I. Yun. A generalized non-linear transformation for evaluating singular integrals. International Journal for Numerical Methods in Engineering, 65(12):1947-1969, 2006.

[45] X.W. Gao and T.G. Davies. Boundary element programming in mechanics. Cambridge University Press, 2002.

[46] G. Beer, I. Smith, and C. Duenser. The boundary element method with programming: for engineers and scientists. Springer, 2008.

[47] Z.R. Niu, W.L. Wendland, X.X. Wang, and H.L. Zhou. A semi-analytical algorithm for the evaluation of the nearly singular integrals in threedimensional boundary element methods. Computer methods in applied mechanics and engineering, 194(9):1057-1074, 2005.

[48] H.L. Zhou, Z.R. Niu, C.Z. Cheng, and Z.W. Guan. Analytical integral algorithm applied to boundary layer effect and thin body effect in BEM for anisotropic potential problems. Computers $\mathcal{E}$ structures, 86(15):1656-1671, 2008. 
[49] H. Ma and N. Kamiya. Distance transformation for the numerical evaluation of near singular boundary integrals with various kernels in boundary element method. Engineering analysis with boundary elements, 26(4):329$339,2002$.

[50] P.R. Johnston and D. Elliott. A sinh transformation for evaluating nearly singular boundary element integrals. International journal for numerical methods in engineering, 62(4):564-578, 2005.

[51] W.J. Ye. A new transformation technique for evaluating nearly singular integrals. Computational Mechanics, 42(3):457-466, 2008.

[52] J.H. Lv, Y. Miao, and H.P. Zhu. The distance sinh transformation for the numerical evaluation of nearly singular integrals over curved surface elements. Computational Mechanics, 53(2):359-367, 2014.

[53] X.Y. Qin, J.M. Zhang, G.Z. Xie, F.L. Zhou, and G.Y. Li. A general algorithm for the numerical evaluation of nearly singular integrals on $3 \mathrm{~d}$ boundary element. Journal of computational and applied mathematics, 235(14):41744186, 2011.

[54] G.Z. Xie, F.L. Zhou, J.M. Zhang, X.S. Zheng, and C.Huang. New variable transformations for evaluating nearly singular integrals in $3 \mathrm{~d}$ boundary element method. Engineering Analysis with Boundary Elements, 37(9):11691178, 2013.

[55] G.Z. Xie, J.M. Zhang, Y.Q. Dong, C. Huang, and G.Y. Li. An improved exponential transformation for nearly singular boundary element integrals in elasticity problems. International Journal of Solids and Structures, 51(6):1322-1329, 2014.

[56] T.A. Cruse. An improved boundary-integral equation method for three dimensional elastic stress analysis. Computers $\mathcal{E}$ Structures, 4(4):741-754, 1974.

[57] T.A. Cruse and R. Aithal. Non-singular boundary integral equation implementation. International journal for numerical methods in engineering, 36(2):237-254, 1993. 
[58] Y.J. Liu and T.J. Rudolphi. Some identities for fundamental solutions and their applications to weakly-singular boundary element formulations. Engineering analysis with boundary elements, 8(6):301-311, 1991.

[59] Y.J. Liu. On the simple-solution method and non-singular nature of the BIE/BEM-a review and some new results. Engineering analysis with boundary elements, 24(10):789-795, 2000.

[60] Y.J. Liu and T.J. Rudolphi. New identities for fundamental solutions and their applications to non-singular boundary element formulations. Сотрutational mechanics, 24(4):286-292, 1999.

[61] E. Klaseboer, C.R. Fernandez, and B.C. Khoo. A note on true desingularisation of boundary integral methods for three-dimensional potential problems. Engineering analysis with boundary elements, 33(6):796-801, 2009.

[62] E. Klaseboer, Q. Sun, and D.Y.C. Chan. Non-singular boundary integral methods for fluid mechanics applications. Journal of Fluid Mechanics, 696:468-478, 2012.

[63] L. Heltai, M. Arroyo, and A. DeSimone. Nonsingular isogeometric boundary element method for Stokes flows in 3D. Computer Methods in Applied Mechanics and Engineering, 268:514-539, 2014.

[64] L. Piegl and W. Tiller. The NURBS book (monographs in visual communication), 1997.

[65] H.J. Kim, Y.D. Seo, and S.K. Youn. Isogeometric analysis for trimmed CAD surfaces. Computer Methods in Applied Mechanics and Engineering, 198(37):2982-2995, 2009.

[66] H.J. Kim, Y.D. Seo, and S.K. Youn. Isogeometric analysis with trimming technique for problems of arbitrary complex topology. Computer Methods in Applied Mechanics and Engineering, 199(45):2796-2812, 2010.

[67] A. P. Nagy and D. J. Benson. On the numerical integration of trimmed isogeometric elements. Computer Methods in Applied Mechanics and Engineering, 284(1):165-185, 2015. 